\title{
Numerical Modelling of an Anodic Metal Bath Heated with an Argon Transferred Arc
}

\author{
Alexandre DOUCE, Clarisse DELALONDRE, Hervé BIAUSSER ${ }^{1)}$ and Jean-Bernard GUILLOT ${ }^{11}$ \\ Fluid Mechanics and Heat Transfer Department, EDF R\&D, 6 quai Watier, BP 49, 78401 Chatou Cedex, France. \\ E-mail: alexandre.douce@edf.fr $\quad$ 1) Laboratoire Elaboration des Matériaux, Ecole Centrale Paris, Grande voie des vignes, \\ 92295 Châtenay-Malabry Cedex, France.
}

(Received on February 26, 2002; accepted in final form on February 4, 2003)

\begin{abstract}
A new 3-D model has been developed to describe the interaction between a transferred electric arc and a liquid metal bath, and has been used to simulate a pilot axisymmetrical transferred arc furnace operating in the EDF Research and Development laboratory.

This model enables calculations of the flow patterns, temperature distribution and electromagnetic fields in both the arc and the bath. The Navier-Stokes equation coupled with the electromagnetic relations are solved in each domain using a finite volume method. The source term in the radiative energy equation is modeled using the radiative transfer method in order to take into account the strong temperature variations in the electric arc. The transport and condensation of metal vapour in the arc domain are considered by solving a conservation equation for the vapour mass fraction. The arc flow calculation at the bath surface uses a one-dimensional sheath model taking account of the metal vapour, in order to ensure the coupling between the plasma and the bath by evaluating the boundary conditions at the arc/bath interface. The calculations were performed for an arc length of $0.25 \mathrm{~m}$.

Realistic predictions are obtained for the electrical, dynamic and thermal behaviour of the plasma and liquid metal, and also for the arc voltage. The results indicate that the effects of the arc impact and Lorentz forces are not sufficient to induce effective mixing throughout the metal bath, leading to a marked thermal stratification in the liquid metal. In the bath, the liquid/solid interface has been determined by calculations.
\end{abstract}

KEY WORDS: steelmaking; tundish; electric arc; argon; numerical simulation; DC arc plasma furnace; argon plasma arc.

\section{Introduction}

More than $80 \%$ of the world's steel is produced by continuous casting processes. The increasing demand for high quality steels requires more accurate control of the liquid steel temperature in the tundish, immediately prior to solidification. Industrial research efforts have attempted to improve this control by the use of additional heating either in the ladle or directly in the tundish. Among the techniques employed, transferred arc plasma heating appears to be one of the most promising. The aim of the present study is to develop a complete model for the heating of an anodic metal bath with a transferred arc plasma torch, in view of potential tundish heating applications.

Present understanding of heat transfer between an electric arc and steel is fairly poor. In order to study this highly complex phenomenon, eight years ago, EDF, the French electricity company, built a pilot reactor, on which numerous temperature and heat flow measurements have been performed during experimental campaigns, enabling the determination of energy balances for the process under steady state conditions. These data have been used to evaluate the new model, which provides a satisfactory description of the reactor behaviour. In a second step, which is outside the scope of the present article, the model will be used by EDF for the design of industrial heating devices.

A transferred arc plasma heating system can be roughly divided into two regions, corresponding to the heating chamber and the liquid bath. The most important zone of the heating chamber is the electric arc. In previous studies, ${ }^{1-8)}$ the arc is often divided into three distinct regions: the arc column and the two near-electrode regions.

The arc column is the largest part of the arc, and links the anode and the cathode. At atmospheric pressure, the plasma is generally collision dominated. The plasma can therefore be viewed as a continuum fluid. Local thermodynamic equilibrium (LTE) and global electrical neutrality are assumed, while a magnetohydrodynamic (MHD) model is often used for the plasma arc column.

Special consideration must be given to the regions near the electrode, where the transition between metallic and gaseous conduction occurs, in order to accurately describe the plasma behaviour. For the anodic region, different more or less sophisticated models exist. The simplest ones merely involve a temperature or current density profile. ${ }^{9)}$ Some use correlations ${ }^{1)}$ to determine heat and mass-transfer between the plasma and the anode. The more sophisticated models are of the anodic layer type, and can take into ac- 
count complex phenomena such as the absence of LTE. ${ }^{10)}$ In the present case, an anodic layer model developed by one of the authors ${ }^{2,3)}$ has been used, which takes into account the presence of metal vapour. In this model, the plasma near the electrode is assumed to be in LTE, since the metal vapour concentration reaches a maximum in this region. ${ }^{4}$

As shown by the recent work of Trenty, ${ }^{5)}$ the other extremely important point in modelling of the heating chamber is the description of radiative heat transfer. Trenty concluded that thermal radiation plays a major role in the heating of both the bath and refractories.

As regards modelling of the metal bath in continuous casting tundish systems, many physical and numerical investigations have been reported in the literature. A review of this work has been made by Mazumdar et al., ${ }^{11)}$ who have shown that classical hydrodynamic models are well adapted for the simulation of liquid metal behaviour. Three dimensional $k-\varepsilon$ turbulent flow models are therefore widely used.

The present study focuses on the interaction between the argon plasma and the iron metal bath, and considers the flow patterns, temperature distributions and electromagnetic fields in the two domains. Particular attention is paid to the effects of radiative heat transfer inside the plasma chamber and to the heat exchanges between the two domains.

\section{Description of the Pilot Arc Furnace}

The reactor geometry considered in the present study is shown in Fig. 1. It is based on an axisymmetrical development furnace supplied by Tetronics to EDF's Plasma Laboratory. This $200 \mathrm{~kW}$ furnace was designed to melt and maintain the temperature of a $300 \mathrm{~kg}$ steel charge. The heating system comprises an argon transferred arc maintained

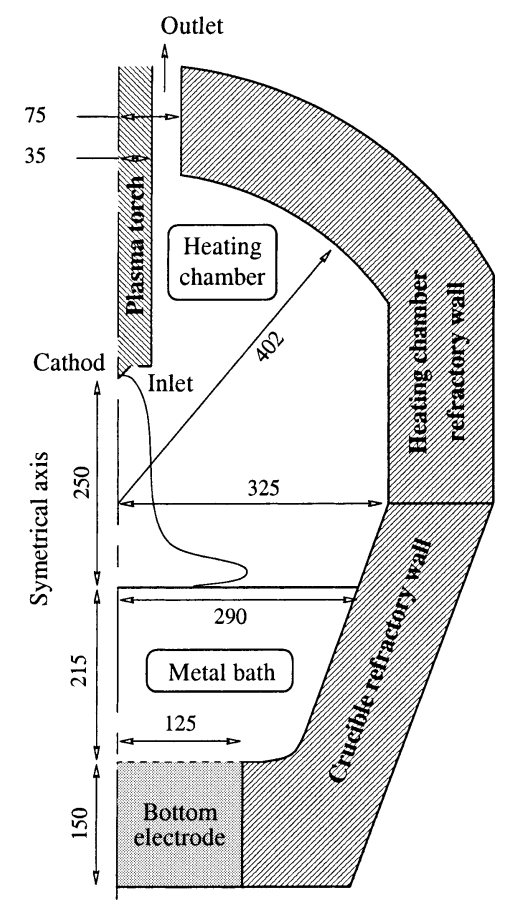

Fig. 1. Oversimplified representation of a half section of the plasma arc furnace. Some characteristic dimensions are reported in $\mathrm{mm}$. between the cathode tip of a $2000 \mathrm{~A}$ plasma torch and a molten iron bath under atmospheric pressure, acting as the anode. The arc length is $250 \mathrm{~mm}$. A $125 \mathrm{~mm}$ radius steel anode is located at the bottom of the furnace. The furnace walls are made of high-grade alumina refractory. The torch body and the refractory walls of the heating chamber are water cooled, while the crucible is air-cooled.

The furnace is instrumented to allow complete thermal monitoring of the process during steady state operation. Thermocouples are embedded in the walls of the furnace and in the bottom electrode to determine the temperatures and heat flows at the internal surfaces using a reverse conduction method. Several tests were carried out under different conditions. The present paper discusses the application of the new model to describe the tests performed using an arc power of $152 \mathrm{~kW}(1750 \mathrm{~A}, 87 \mathrm{~V})$.

\section{Numerical Model}

\subsection{Basic Features and Assumptions of the Model}

As shown in Fig. 2, for computing purposes, the furnace geometry is divided into three sub-domains, corresponding to the plasma, the metal bath, and the anodic layer. The metal bath and the bottom electrode are treated simultaneously in the same calculation domain. The liquid/solid interface is not fixed but it is a result of the calculations.

The model focuses on the radiative heat balance in the heating chamber, in order to evaluate the contribution of the arc radiation, particularly at the furnace walls. An accurate description of radiative heat transfer imperatively requires the use of a 3-D model, even though the pilot furnace geometry and then the plasma flow are axisymmetrical. Each calculation sub-domain is therefore represented by a 3-D angular sector, delimited by two planes of symmetry located an each end of the angular sector: this geometry is a better arrangement than the use of a complete 3-D domain,

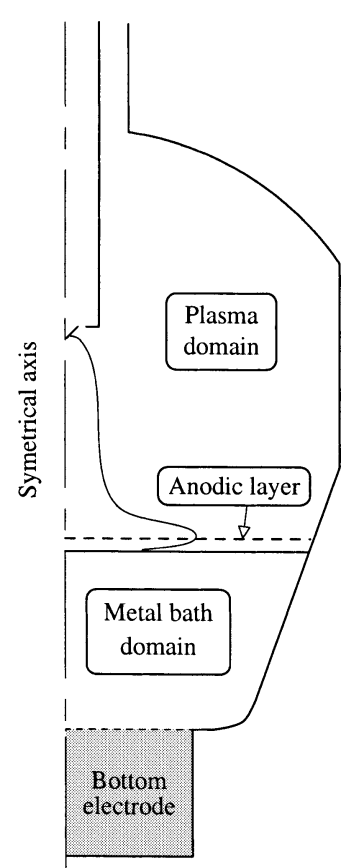

Fig. 2. Calculation sub-domains. In the metal bath domain, the liquid and solid phases are treated together. 
in order to reduce the CPU time. The plasma domain is divided into 37095 cells, and the metal bath domain into 29890 cells, the mesh being finer at the plasma/metal interface to allow a detailed description of plasma impact effects. Overall views of the mesh used are shown in Figs. 3 and 4: the symmetry planes are. In order to precisely describe the interface, the anodic layer is treated with a local one-dimensional subgrid.

The formulation of the model is based on the following assumptions:

(1) Steady state flow conditions are considered in both the plasma and metal domains. A low Reynolds number $k-\varepsilon$ turbulent flow model is used for the liquid phase. But as it was pointed out in previous work ${ }^{5}$ using the low Reynolds $k-\varepsilon$ model, the flow is found to be laminar in the plasma gas domain. LTE is assumed. The arc column is described by a MHD model, considering the conservation laws for momentum, mass and energy, together with the equation of state and simplified Maxwell equations. Considering the rather low current intensity of our arc, and by consequence the low intensity of the magnetic field, the simplified Ohm law is available in our simulations. ${ }^{8)}$ Although the transient flow behaviour is not the principal aim of the model, the conservation laws are written with their unsteady term,

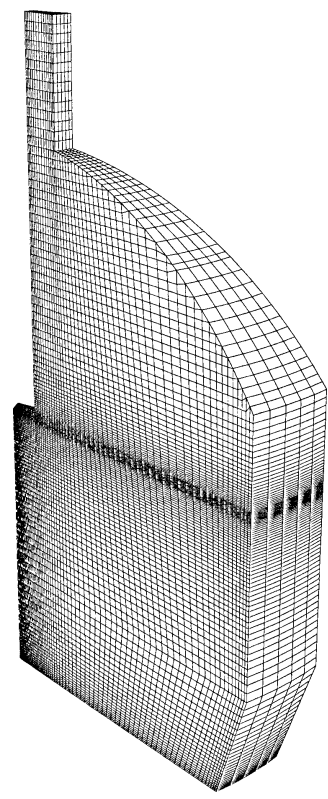

Fig. 3. Calculation mesh for the heating chamber (plasma domain).

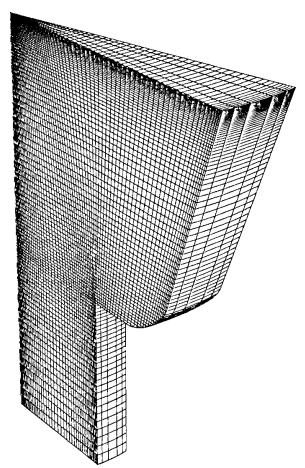

Fig. 4. Calculation mesh for the liquid bath (metal bath domain). since the discretization of the transient equations is analogous to that of the under-relaxed steady state equations.

(2) Iron evaporation effects in the plasma chamber and at the plasma/metal bath interface are included. The thermodynamic properties and transport coefficients of the gas depend on its temperature and composition (iron vapour content). The iron vapour condensation on the walls and in the plasma chamber is taken into account by assuming that the phase transition occurs at a constant temperature, but its contribution to the energy balance is neglected. It is assumed that the condensation occurs when the partial pressure of the iron vapour is larger than the saturated vapour pressure. ${ }^{12)}$ In this case, a new iron vapour concentration is estimated by using this saturated vapour pressure.

(3) There is no special treatment of the cathodic region, since the energy exchanges in the cathodic layer are not the aim of the present study. In contrast, specific attention is paid to modelling of the anodic layer in order to accurately describe the heat and mass transfer in this region. Because of the presence of metal vapour, LTE is assumed to prevail in the anodic layer. Moreover, no interface deformation is considered, the bath surface being assumed to remain flat. Marangoni forces are not considered in this study ${ }^{5)}$ : this assumption will be confirmed by the results, and by the fact that the temperature in the bath is fairly uniform in the upper zone.

\subsection{Governing Equations}

\subsubsection{Plasma Domain}

The plasma is a gaseous mixture of argon and metal vapour. It is treated as a continuous fluid using the classical fluid mechanics equations, with the contribution of Lorentz forces in the momentum equation, and resistance heating and radiative source terms in the energy equation. The mass and momentum conservation equation can be written:

$$
\frac{\partial \rho}{\partial t}+\vec{\nabla} \cdot(\rho \vec{u})=0
$$

and

$$
\rho\left(\frac{\partial \vec{u}}{\partial t}+\vec{u} \cdot \vec{\nabla} \vec{u}\right)=-\vec{\nabla} p+\rho \vec{g}+\vec{\nabla} \cdot \vec{\tau}+\vec{J} \wedge \vec{B}
$$

where, $\rho, \vec{u}, p, \vec{g}, \vec{\tau}, \vec{J}$, and $\vec{B}$ represent respectively the density, velocity, pressure, gravitational acceleration, viscous stress tensor depending on the plasma viscosity plotted on Fig. 5, current and magnetic field. The current $\vec{J}$ and the magnetic field $\vec{B}$ induced by the current define the Lorentz forces $\vec{J} \wedge \vec{B}$.

The conservation of energy, formulated in terms of enthalpy, can be expressed as:

$$
\rho\left(\frac{\partial h}{\partial t}+\vec{u} \cdot \vec{\nabla} h\right)=\vec{\nabla} \cdot\left(\frac{\lambda_{\text {gas }}}{C_{\mathrm{p}}} \vec{\nabla} h\right)+\frac{J^{2}}{\sigma_{\text {gas }}}+S_{\text {rad }} \ldots \ldots(3)
$$

where $h$ is the enthalpy, $\lambda_{\text {gas }}$ the thermal conductivity of the gaseous mixture see Fig. 6, $C_{\mathrm{p}}$ the specific heat, $\sigma_{\text {gas }}$, see Fig. 7, is the electrical conductivity in the expression for the resistance heating source terms $J^{2} / \sigma_{\text {gas }}$, and $S_{\text {rad }}$ is the radiative heat loss term, whose calculation is explained below. This equation supposes that the diffusion coeffi- 


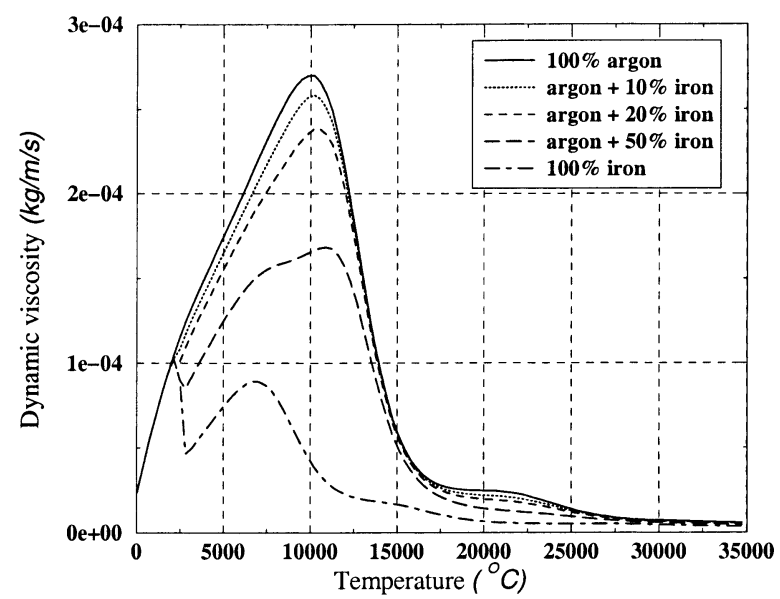

Fig. 5. Dynamic molecular viscosity of an argon plasma with iron vapour.

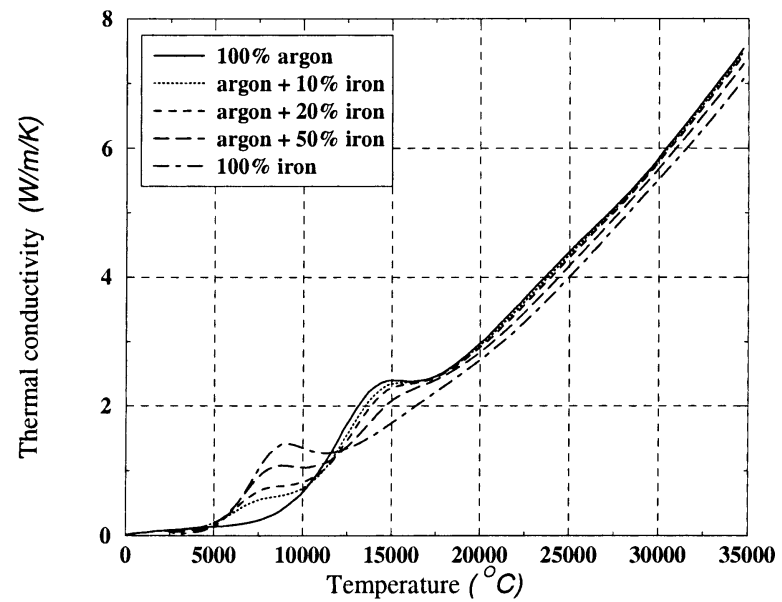

Fig. 6. Thermal conductivity of an argon plasma with iron vapour.

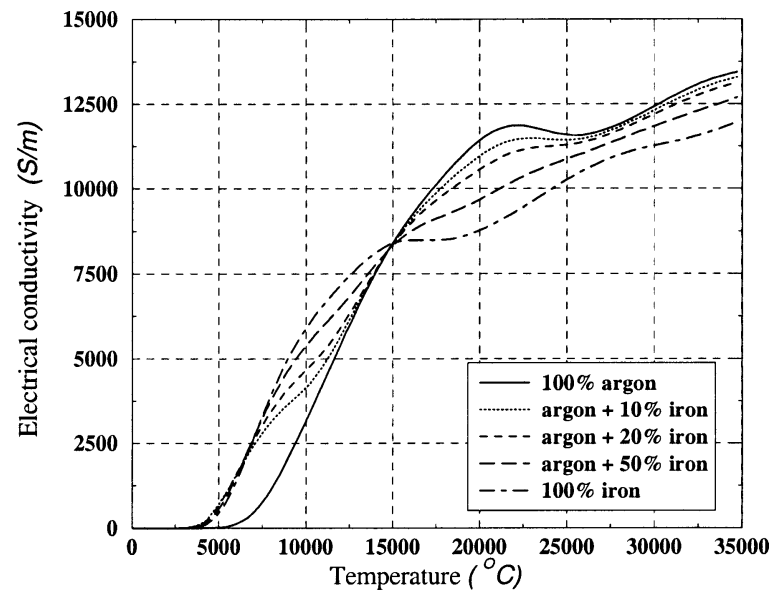

Fig. 7. Electrical conductivity of an argon plasma with iron vapour.

cients and thermal diffusivities are the same for the different species. The transport of electron enthalpy due to electron drift is not considered in this formulation, since previous work ${ }^{5)}$ has shown it to be negligible.

These equations are then coupled with the electromagnetic relations. Using the electrical potential $\Phi$, defined as $\vec{J}=\sigma_{\text {gas }} \vec{\nabla} \Phi$, the current conservation criterion can be writ- ten:

$$
\vec{\nabla} \cdot\left(\sigma_{\text {gas }} \vec{\nabla} \Phi\right)=0
$$

Due to the axisymmetrical geometry, the magnetic field $B V$ ${ }_{\theta}$ is calculated directly from the current density distribution using Ampere's theorem:

$$
B_{\theta}(R, \theta, z)=\int_{0}^{R} J_{z}(R, \theta, z) r d r
$$

In addition, a conservation equation for iron is solved:

$$
\rho\left(\frac{\partial X_{\mathrm{Fe}}}{\partial t}+\vec{u} \cdot \vec{\nabla} X_{\mathrm{Fe}}\right)=\vec{\nabla} \cdot\left(\rho D_{\mathrm{Fe}} \vec{\nabla} X_{\mathrm{Fe}}\right)
$$

where $X_{\mathrm{Fe}}$ is the iron concentration and $D_{\mathrm{Fe}}$ is the diffusion coefficient.

All physical properties of the gaseous mixture used in these equations, were computed specifically for the present study. ${ }^{13)}$

For the 3-D modelling of radiative heat transfer in the plasma chamber, a discrete ordinate method, developed for finite volumes, has been implemented. ${ }^{14)}$ The radiative source term $S_{\text {rad }}$ calculation is based on the following expression:

$$
S_{\mathrm{rad}}=-\vec{\nabla} \cdot\left(\int_{0}^{4 \pi} I \vec{S} d \Omega\right)
$$

where $d \Omega$ is the elementary solid angle around the radiative propagation direction $\vec{S}$ and $I$ is the intensity of radiation. $I$ is obtained from the radiative heat transfer equation for grey semi-transparent non-diffusive media:

$$
\vec{\nabla} \cdot(I \vec{S})=-K I+K I^{\mathrm{b}}
$$

where $K$ is the absorption coefficient of the gas, and $I^{\mathrm{b}}$ the black body intensity.

\subsubsection{Metal Bath Domain}

The heat and fluid flows require the same set of conservation equations as for the plasma domain. However, in the metal bath, turbulence must be taken into account. Indeed, due to the large variations in the molecular viscosity, both laminar and turbulent regimes may be present in the metal flow. This is allowed for in the present study by means of a two-equation low Reynolds number $k-\varepsilon$ model. ${ }^{15,16)}$ The possible effect of turbulence on the electrical conductivity is neglected.

Because of the magnetic field generated by the electric arc, the effects of Lorentz forces and resistance heating are taken into account in the momentum and energy conservation laws. The metal domain therefore requires the same set of governing electromagnetic equations as the plasma domain.

\subsection{Boundary and Interface Conditions}

\subsubsection{Plasma Domain}

At the inlet, a $6 \mathrm{~m}^{3} \cdot \mathrm{h}^{-1}$ argon flow is imposed. The gas outlet is considered as an open boundary. In order to evaluate the temperature $T_{\mathrm{w}}$ at the refractory and plasma torch 
walls, an important aspect of the simulation is to determine the radiative flux density at these points. The incident radiative flux density on the walls is given by:

$$
Q_{\text {incid }}=\int_{0}^{2 \pi} I|\vec{S} \cdot \vec{n}| d \Omega
$$

where $\vec{n}$ refers to the wall normal and $\vec{S}$ is the radiative propagation direction from the medium to the wall. The heat balance at the wall is then improved by considering the heat exchanges as follows:

$$
-\frac{\lambda_{\mathrm{w}}}{e}\left(T_{\mathrm{w}}-T_{\mathrm{ext}}\right)+\varepsilon Q_{\mathrm{incid}}=h_{\mathrm{gas}}\left(T_{\mathrm{w}}-T_{\mathrm{gas}}\right)+\varepsilon \sigma T_{\mathrm{w}}^{4}
$$

where $\lambda_{\mathrm{w}}, e$ and $\varepsilon$ represent the thermal conductivity, thickness and emissivity of the furnace walls, $\sigma$ is the Stefan-Boltzmann constant, $T_{\text {ext }}$ the external temperature of the furnace, $T_{\text {gas }}$ the temperature of the gaseous mixture and $h_{\text {gas }}$ is the heat transfer coefficient between the gas and the walls, coming from the modelling of the turbulent transfer in the boundary layer that occurs in ESTET code $^{16)}$ : in the case of a laminar flow $h_{\text {gas }}$ depends only to molecular thermal conductivity of the plasma gas. The refractory temperature is deduced from Eq. (10).

At the cathode tip, the normal temperature derivative and electrical potential are taken to be zero. The potential gradient at the bath surface is assumed to be low, so that the potential is uniform. The heat flux density and the iron vapour flux density at the anodic surface are imposed, being determined by the anodic layer calculation domain.

\subsubsection{Metal Bath Domain}

At the bath surface, the heat flux density and the normal current density are determined by the anodic layer domain, and are thus imposed, together with the surface shear stress distribution calculated from the plasma domain. At the bottom electrode, the temperature is taken as $500^{\circ} \mathrm{C}$, which is the value measured on the experimental installation. On the lateral surface of the bottom electrode, zero normal derivatives are imposed for both the temperature and the current density.

\subsubsection{Anodic Layer Domain}

The anodic layer is a thin plasma layer where processes are complex and diverse. Here, a model based on the presence of the metallic vapour in the anode region, is proposed. ${ }^{2)}$ Following this model, the current transfer in the anode layer is partially ensured by the free electrons coming from the ionization of the metallic vapour which have considerably lower ionization energy than the working gas.

It is assumed that the plasma near the anode is in LTE and composed by free electrons, argon species and iron ones. Further it is assumed that the partial pressure of the metallic vapour at the boundary is a function of the local temperature according to liquid-vapour transition curve. In this anode region, the radial gradients are negligible compared to the gradients along the axis of the arc. This mean that the thickness of the region is very small than the radius of the arc. Under these assumptions, the problem can be simplified to a one-dimensional in the normal direction to the anode surface, say $z$, where the following equations are solved:

$$
\begin{aligned}
& \frac{\partial J_{z}}{\partial z}=0 \\
& \frac{\partial \rho u_{z}}{\partial z}=0 \\
& \rho u_{z} \frac{\partial h}{\partial z}=\frac{\partial}{\partial z}\left(\frac{\lambda_{\mathrm{gas}}}{C_{\mathrm{p}}} \frac{\partial h}{\partial z}\right)+\frac{5}{2} J_{z} \frac{k_{\mathrm{B}}}{q C_{\mathrm{p}}} \frac{\partial h}{\partial z}+\frac{J_{z}^{2}}{\sigma_{\mathrm{gas}}} \\
& \rho u_{z} \frac{\partial X_{\mathrm{Fe}}}{\partial z}=\frac{\partial}{\partial z}\left(\rho D_{\mathrm{Fe}} \frac{\partial X_{\mathrm{Fe}}}{\partial z}\right)
\end{aligned}
$$

$q$ is the electronic charge, $k_{\mathrm{B}}$ is Boltzmann constant.

The present model describes the temperature and iron mass concentration distributions in the anodic layer, and ensures the electrical and thermal coupling between the arc plasma and the metal bath surface, and then induces the boundary conditions for the concentration of the vapour in the arc.

The boundary conditions for the anodic layer domain are determined by the plasma and bath domains, as shown in Fig. 8.

At the upper edge of the anodic layer, the temperature, the iron mass fraction and the current density resulting from the 3-D calculation are imposed. At the anodic wall, the metal bath surface is imposed. The iron mass fraction is imposed and calculated as a function of the temperature on the bath surface and the iron saturated vapour pressure.

The heat flux transmitted to the metal bath is evaluated from the enthalpy balance on the negative space charge zone near the anode surface as follows:

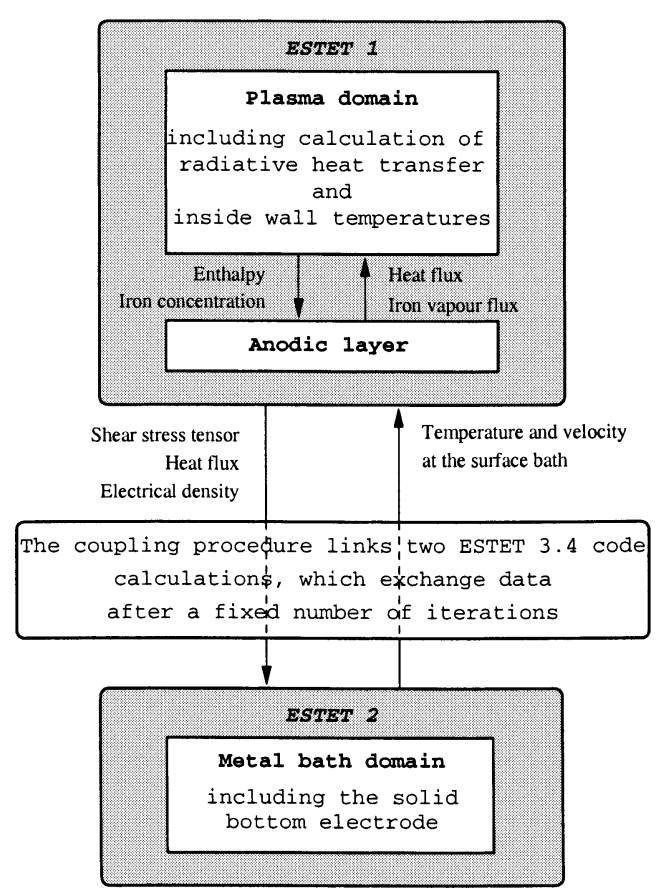

Fig. 8. Schematic numerical procedure for coupling the two calculation domains. 


$$
\lambda_{\mathrm{Fe}} \frac{\partial T_{\mathrm{Fe}}}{\partial z}=\lambda_{\mathrm{gas}} \frac{\partial T_{\mathrm{gas}}}{\partial z}+j\left(V_{\mathrm{a}}+\Phi_{\mathrm{a}}\right)-L_{\mathrm{Fe}} \Phi_{\mathrm{v}}
$$

where $\lambda_{\mathrm{Fe}}$ is the thermal conductivity for iron. $V_{\mathrm{a}}, \phi_{\mathrm{a}}$ and $L_{\mathrm{Fe}}$ are the anode fall set at $3.5 \mathrm{~V}$, the work function of the anode material equal to $4.5 \mathrm{~V}$, and the latent heat of vaporisation of iron. ${ }^{12)}$ The study of the arc-anode interactions needs the knowledge of $\Phi_{\mathrm{v}}$, the rate of vaporisation or condensation of the electrode material. This rate has to consider the background pressure, the anode material and the convection and diffusion of the vapour in the surrounding plasma. In the present work, the vaporisation rate is given by:

$$
\Phi_{\mathrm{v}}=\frac{1}{\left(1-\left(X_{\mathrm{Fe}}\right)_{0}\right)} \rho_{0}\left(D_{\mathrm{Fe}}\right)_{0}\left(\frac{\partial}{\partial z} X_{\mathrm{Fe}}\right)_{0}
$$

The subscrip ' 0 ' tin the above equation indicates the values of the variables in the first point near the bath surface.

\subsection{Numerical Method}

The calculations were performed using the commercial software ESTET 3.4, a 3-D finite volume fluid dynamics with quick-upwind differencing scheme, code developed by EDF for single bloc structured half-staggered grid. ${ }^{16)}$

The calculation domain is divided into three parts, corresponding to the plasma flow, the metal bath and the anodic region. These different domains are treated separately, and a numerical procedure is then used to link them together. This coupling procedure is based on the equivalence of boundary exchanges, between the anodic layer and the arc column, and between the anodic layer and the free surface of the metal bath, as described in the preceding paragraphs.

The anodic layer and the plasma domain are treated simultaneously, in order to modify the boundary conditions for the plasma arc column at each time step, taking into account the metal vapour concentration (Fig. 8).

The mass conservation and momentum equations are solved using the modified SIMPLEC algorithm with strong pressure velocity-coupling. The energy equation and the electron conservation equations are solved using a finite volume method. In the solid metal domain, the viscosity is taken as $10^{8} \mathrm{~kg} \cdot \mathrm{m}^{-1} \cdot \mathrm{s}^{-1}$ in order to ensure the absence of flow.

Computations were performed on a CRAY YMP, and needs several tens of hours of CPU time. The time step has been set at $10^{-7} \mathrm{~s}$ for each iteration in the plasma domain and at $10^{-3} \mathrm{~s}$ in the bath.

\section{Results and Discussion}

Calculations were performed for a $1750 \mathrm{~A}$ arc in argon at atmospheric pressure. The results are compared to experimental data obtained in the pilot arc furnace, under the same operating conditions. A selection of computed results are discussed below, paying special attention to the metal bath behavior and the furnace heat balance. In the plasma domain, we have only plotted the iron vapour mass fraction on Fig. 9.

The iron vapour, generated in the anodic layer, reaches its maximum value near the metal bath, and are then carried away by the plasma flow towards the plasma chamber inner

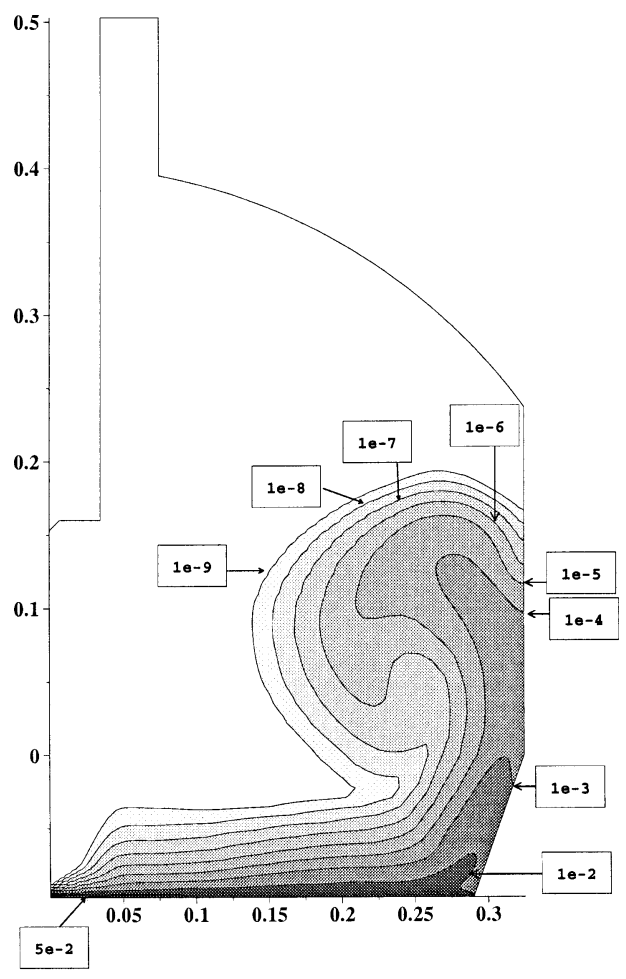

Fig. 9. Calculated iron mass fraction in the plasma domain.

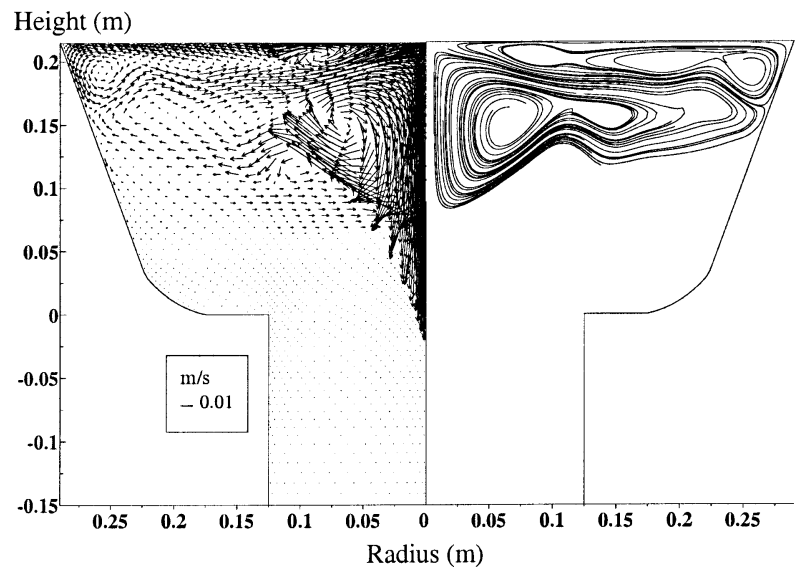

Fig. 10. Predicted flow patterns and streamlines in the central vertical plane of the metal bath calculation domain.

walls. These values of iron vapour have a great influence of the thermal properties of the plasma (see Figs. 5, 6 and 7), especially on the electrical conductivity: the metallic vapour ensures the electrical conduction.

Figure 10 represents the velocity vectors and streamlines in the metal bath. The flow is characterized by two recirculatory zones (vortices). The first recirculatory flow close to the bath surface, is driven by the plasma flow in an outward direction. The arc plasma jet impacting on the bath and then going to the chamber wall induces tangential shear stresses that cause this recirculatory flow just beneath the interface. The second recirculatory flow is directed inward under the free surface, located near the axis of the calculation domain, and is due to electromagnetic forces. These electromagnetic forces ensure better mixing of the metal in this region. The maximum liquid metal velocity is 0.2 $\mathrm{m} \cdot \mathrm{s}^{-1}$ and occurs near the axis of the bath calculation do- 


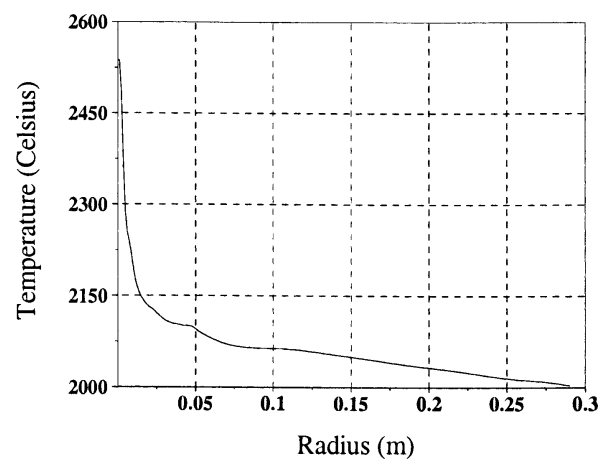

Fig. 11. Free surface temperature in ${ }^{\circ} \mathrm{C}$.

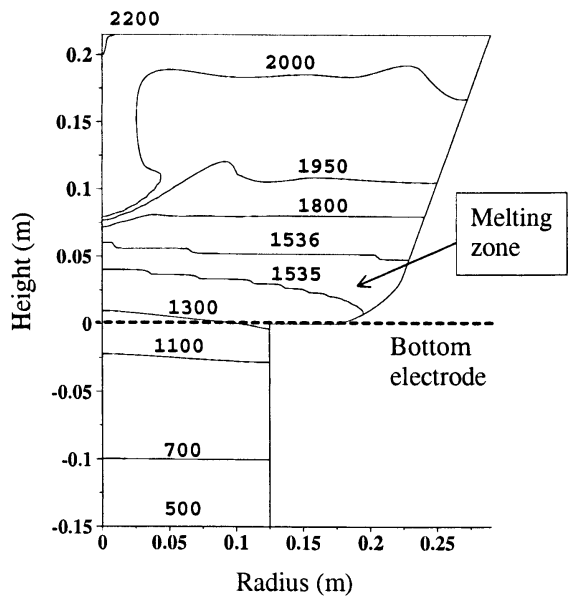

Fig. 12. Metal bath isotherms in ${ }^{\circ} \mathrm{C}$. The melting zone is assumed to be situated between 1535 and $1536^{\circ} \mathrm{C}$.

main. The order of magnitude of this result is close to the values reported for previous work on molten iron and steel baths. ${ }^{3,5,11)}$

The temperature profile at the free surface, plotted in Fig. 11, shows that the liquid metal is superheated just beneath the arc zone, reaching a temperature close to $2500^{\circ} \mathrm{C}$ on the furnace axis. Because of the mixing effect due to electromagnetic forces, the temperature in the bath is fairly uniform in the upper zone, up to the $1950^{\circ} \mathrm{C}$ isotherm, as shown in Figs. 12 and 13, corresponding respectively to the isotherms in the metal bath and the temperature profile along the axis of the calculation domain.

In contrast, between the 1950 and $1536^{\circ} \mathrm{C}$ isotherms, the temperature homogeneity in the bath is poor, with a marked thermal stratification. Indeed, the mixing effect, due to both the arc impact and Lorentz forces, is not sufficient to ensure good thermal homogeneity throughout the bath (see also Fig. 10).

The solidification zone is rather large and is located between 1535 and $1536^{\circ} \mathrm{C}$. Near the bottom electrode, there is a region of solid metal, below $1535^{\circ} \mathrm{C}$, larger than the electrode itself. This result indicates that the calculations probably overestimated the heat losses at the crucible refractories. The axial temperature profile in Fig. 13 clearly reveals the solid zone, corresponding to a linear variation of temperature between 500 and $1535^{\circ} \mathrm{C}$, followed by the constant temperature melting zone. Beyond the latter, a further steep linear rise in temperature is observed, in the region where the metal is virtually stagnant. This region corre-

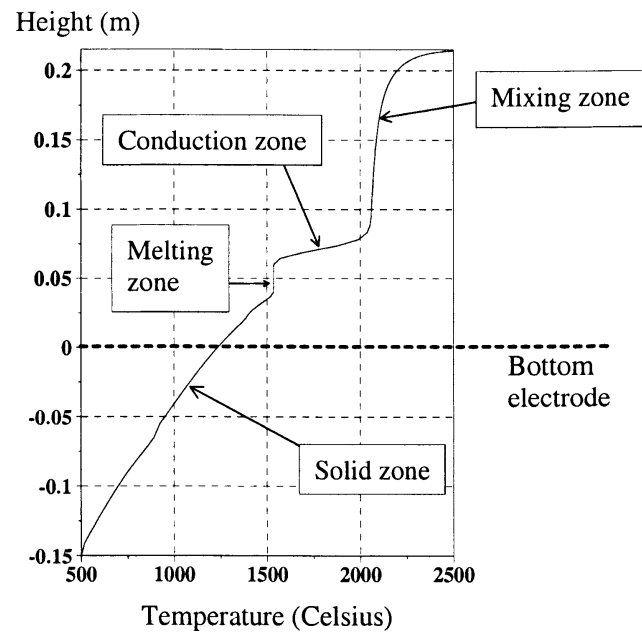

Fig. 13. Metal bath axial temperature profile in ${ }^{\circ} \mathrm{C}$.

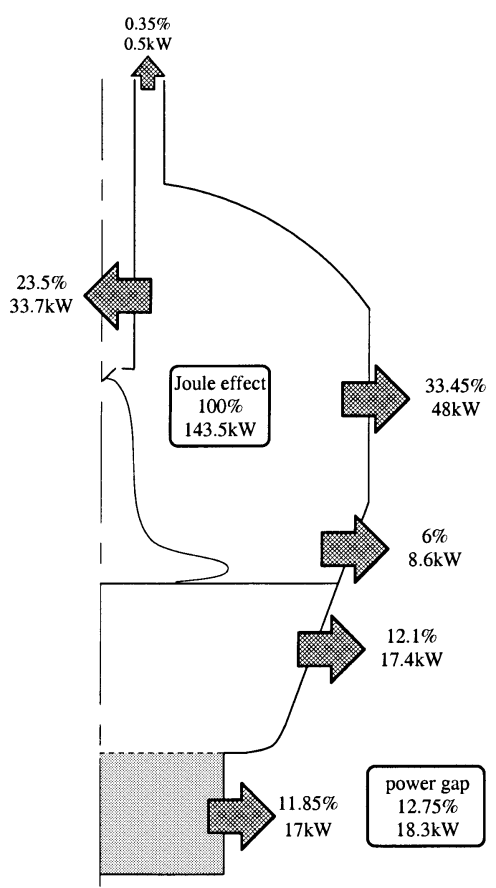

Fig. 14. Numerically predicted energy flow sheet.

sponds to a thermal conduction zone.

The calculated arc voltage $(82 \mathrm{~V})$, is in good agreement with the measured value $(87 \mathrm{~V})$, justifying the assumption of a zero voltage drop across the cathode sheath. The electrical power supply was therefore evaluated to be $143.5 \mathrm{~kW}$.

Figure 14 illustrates the heat balance in the torch, the heating chamber and the crucible refractories. The energy transferred from the plasma to the crucible and the bottom electrode represents $29.9 \%$ of the total electrical power supply, corresponding to an overestimation compared to the measured value, reported in Table 1. Concerning the energy exchange between plasma and bath domains are detailed on the next figure.

Indeed, the computed heat balance for the furnace is not sufficiently realistic at this stage of the work, for two reasons. Firstly, the boundary conditions for the radiative heat transfer equation are represented by the symmetry planes, which are treated as fully non-directional reflection planes. Secondly, the difference between the electrical power con- 
Table 1. Comparison between calculated and measured heat losses in the pilot furnace.

\begin{tabular}{ccc}
\hline & $\begin{array}{c}\text { Experimental } \\
\text { data }\end{array}$ & $\begin{array}{c}\text { Calculation } \\
\text { results }\end{array}$ \\
\hline Plasma torch & $36.7 \%$ & $23.5 \%$ \\
\hline Heating chamber & $64.95 \mathrm{~kW}$ & $48 \mathrm{~kW}$ \\
refractories & $42.66 \%$ & $33.45 \%$ \\
\hline Crucible & $26.83 \mathrm{~kW}$ & $43 \mathrm{~kW}$ \\
refractories and & $17.62 \%$ & $29.95 \%$ \\
bottom electrode & & \\
\hline
\end{tabular}

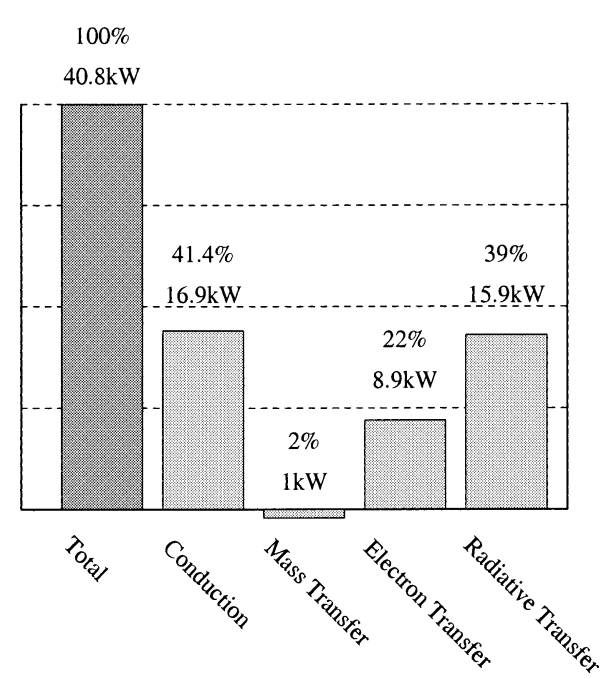

Fig. 15. Thermal contributions to metal bath heating calculated using the anodic layer model.

sumed and the heat losses from the furnace walls is close to $12.7 \%$. This discrepancy is due to the numerical coupling of the calculation domains and is extremely difficult to reduce.

The different components of the heat flux transferred from the arc plasma to the metal are plotted in Fig. 15. It can be seen that thermal conduction and radiative heat transfer, each of similar magnitude, represent the largest contributions. The effect of mass transfer (evaporation of the metal beneath the arc zone) is quite negligible.

\section{Conclusions}

A new fluid dynamics model has been developed to describe the interaction between an anodic metal bath and a transferred arc plasma torch, for potential application to tundish heating systems. Numerical simulations have been carried out using the ESTET 3.4 code and a 3-D finite volume software, developed by EDF Research and Development.

The original feature of the work is the specific treatment of the anodic layer which ensures the electrical, thermal, and dynamic coupling between the plasma and the metal domains. In order to improve the heat flow in the reactor, special attention has been paid to modelling of radiative heat transfer in the plasma chamber.

At the present stage of the work, the results obtained when the model is applied to a pilot arc furnace are quite realistic as regards the dynamic and thermal behaviour in the plasma and metal bath domains. The simulations show that total melting of the metal is not obtained in the crucible, in good agreement with experiments. In the experimental configuration used, the lower part of the metal is not melted, probably due to the excessive size of the bottom electrode, which entrains large energy losses and marked temperature gradients. One important result of the simulations is to suggest a new design for the bottom electrode to ensure more efficient furnace operation.

A significant improvement of the model is the introduction of a 3-D description of radiative heat transfer. Further development of the model will require better treatment of the boundary conditions for radiative heat transfer, in the case of a symmetrical approach, together with more accurate knowledge of the radiative properties of the plasma and the thermal properties of the refractories. The latter point is extremely important, and specific measurements or parametric numerical studies would be of great interest to enable further improvement of the results. Ultimately, the introduction of a non-axisymmetrical electrical potential would allow fully 3-D calculations.

Finally, it has been shown that the ESTET calculation code is capable of performing complex physical simulations, not only in the field of plasma and electric arc applications, but also in the case of melting processes including both liquid and solid zones.

\section{REFERENCES}

1) L. Gu: Doctorate Thesis, Norwegian Inst. of Tech., Trondheim, (1993).

2) A. Kaddani, C. Delalondre, O. Simonin and H. Minoo: High Temp. Chem. Processes, 3 (1994), 411.

3) L. Trenty, A. Bouvier, C. Delalondre, O. Simonin and J. B. Guillot: Proc. 4th Int. Thermal Plasma Processes Conf., Begell House, Athens, (1996), 527.

4) J. J. Gonzalez, A. Gleizes, P. Proulx and M. Boulos: J. Appl. Phys., 74 (1993), 3065.

5) L. Trenty: Doctorate Thesis, Ecole Centrale Paris, (1997).

6) J. J. Lowke, R. Morrow and J. Haidar: J. Phys. D: Appl. Phys., 30 (1997), 2033.

7) S. Paik and D. H. Nguyen: Int. J. Heat Mass Transfer, 38 (1995), 1161.

8) A. Douce: Doctorate Thesis, Ecole Centrale Paris, (1999).

9) K. C. Hsu, K. Etemadi and E. Pfender: J. Appl. Phys., 54 (1983), 3818.

10) H. A. Dinulescu and E. Pfender: J. Appl. Phys., 51 (1980), 3149.

11) D. Mazumdar and R. I. L. Guthrie: ISIJ Int., 39 (1999), 524.

12) T. Yoshida and K. Akashi: J. Appl. Phys., 48 (1977), 2252.

13) B. Chervy and A. Gleizes: Calcul des propriétés des mélanges Argon-Fer à la pression atmosphérique, EDF-CPAT Internal Report, (1996).

14) W. A. Fiveland: J. Heat Transfer, 106 (1984), 699

15) B. E. Launder and B. I. Sharma: Letters Heat Mass Transfer, 1 (1974), 131.

16) J. D. Mattéi and O. Simonin: Logiciel ESTET - Manuel thériqueTome 1: Modéisations physiques, EDF Internal Report HE44/92/38/B, (1993). 